\title{
Optimising solvent extraction for metal recycling
}

The platinum-group metals play a central role in several technological applications including fuel cells, electronic devices, and catalysts for oil refining and automobile of these metals methods for their recycling from end-oflife devices are in increasing demand. Hydrometallurgy, which exploits the ability of specific molecules to selectively dissolve metal ions and separate them from unwanted species, metal recycling. The efficioncy of extractants in solvent extraction, however are often assessed using model solutions, rather than real metal-rich aqueous phases. Assistant Professor Ana Paula Paiva, from the University of Lisbon, Portugal, shows that the development of efficient new extractants of their performance on real spent catalysts.

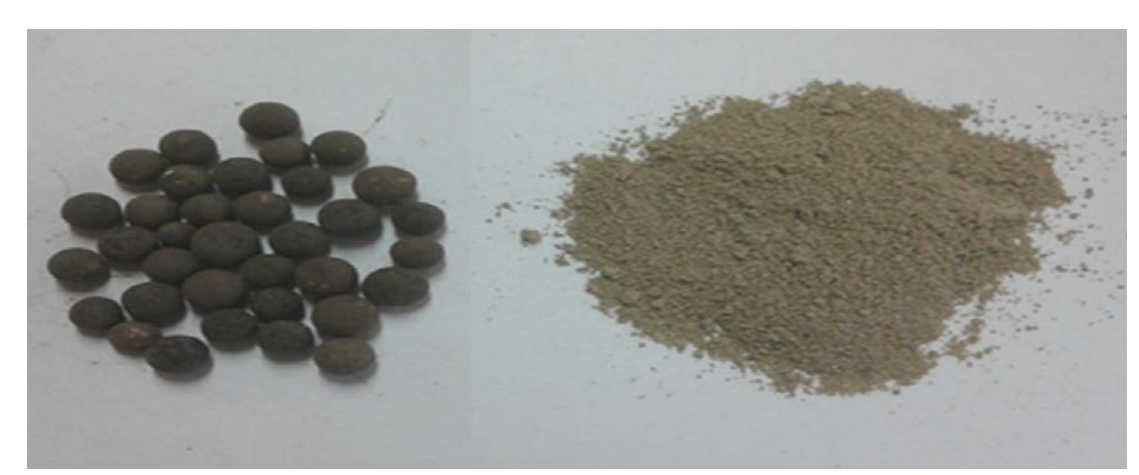

A spent palladium catalyst from a Portuguese petrochemical industry, used to hydrogenate
methylacetylene and propadiene to propylene and propane

of six precious elements. Clustered together in the Periodic Table, they include platinum, palladium, and hodium. These metals have similar exhibit high resistance to chemical excellent mechanical and electrical properties, and good ductility They are also highly resistant to water and tarnish, which makes them - particularly in the case of platinum - ideally suited for fine jewellery. However, their chemical activity is the main factor that explains the technology and the consequent rapid increase in demand for them.

\section{CATALYSIS}

Platinum-group metals can act as catalysts, promoting chemical reactions that would not normally occur spontaneously, and they are therefore industrial-scale chemical proceses, oil refining, anti-cancer drugs, and in the development of electronic devices. An essential and ubiquitous example of thei use is in catalytic converters for exhaust emission control in automobiles. In this

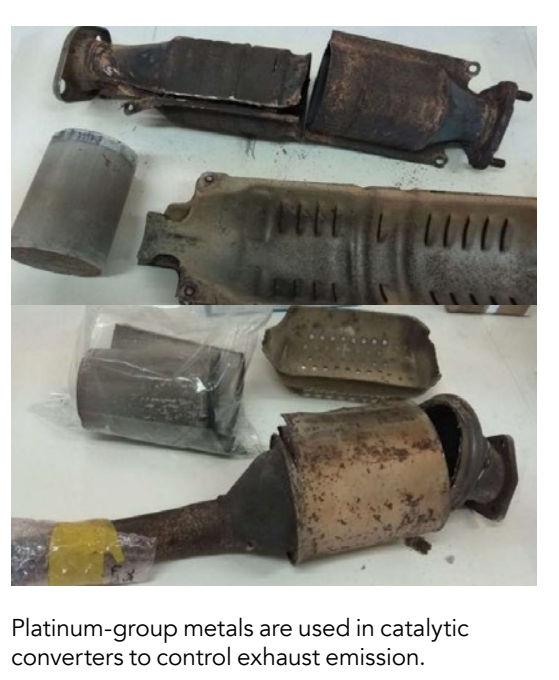

case, platinum, palladium, and rhodium are the active chemical species that convert harmful exhaust gases (including carbon monoxide, nitric oxide, nitric dioxide, and hydrocarbons) into far less dioxide, molecular nitrogen, and water.

\section{RARE AND PRECIOUS RESOURCES}

The mineral deposits of platinum-group metals on the Earth's surface are very limited and unevenly distributed, with South Africa and Russia being virtually the only primary producers of platinum, palladium, and rhodium. This explains the high value and cost of these elements and poses severe constraints on their availability as critical raw materials for technology. To avoid ore exhaustion and prevent the irreversible environmenta damage caused by large-scale metal extraction, the development of affordable and sustainable recycling protocols to extract these precious species from enda pressing priority.

\section{METAL EXTRACTION}

There are two main methods for

separating metals from ores or end-of-life terials. One class of techniques, known as pyrometallurgy, uses high temperatures has been known and extensively used for millennia. By contrast, hydrometallurgy is a comparatively newer technique, which exploits aqueous chemistry to achieve the same goal. Pyrometallurgy has higher energy consumption and potentially high metal loss and can create severe environmental hazards, owing to the generation of gases and dust during the extraction process. In hydrometallurgy, the energetic and environmental costs are far reduced. In this case, however, specific chemicals, solvents, and separation optinised mus be developed and optinised to achieve a satisfactory metal

\section{TARGETING METAL RECOVERY}

The work of Assistant Professor Ana devoted to advancing hydrom $L$ Lisbon is techniques, with the aim of optimising them for the extraction of platinum-group

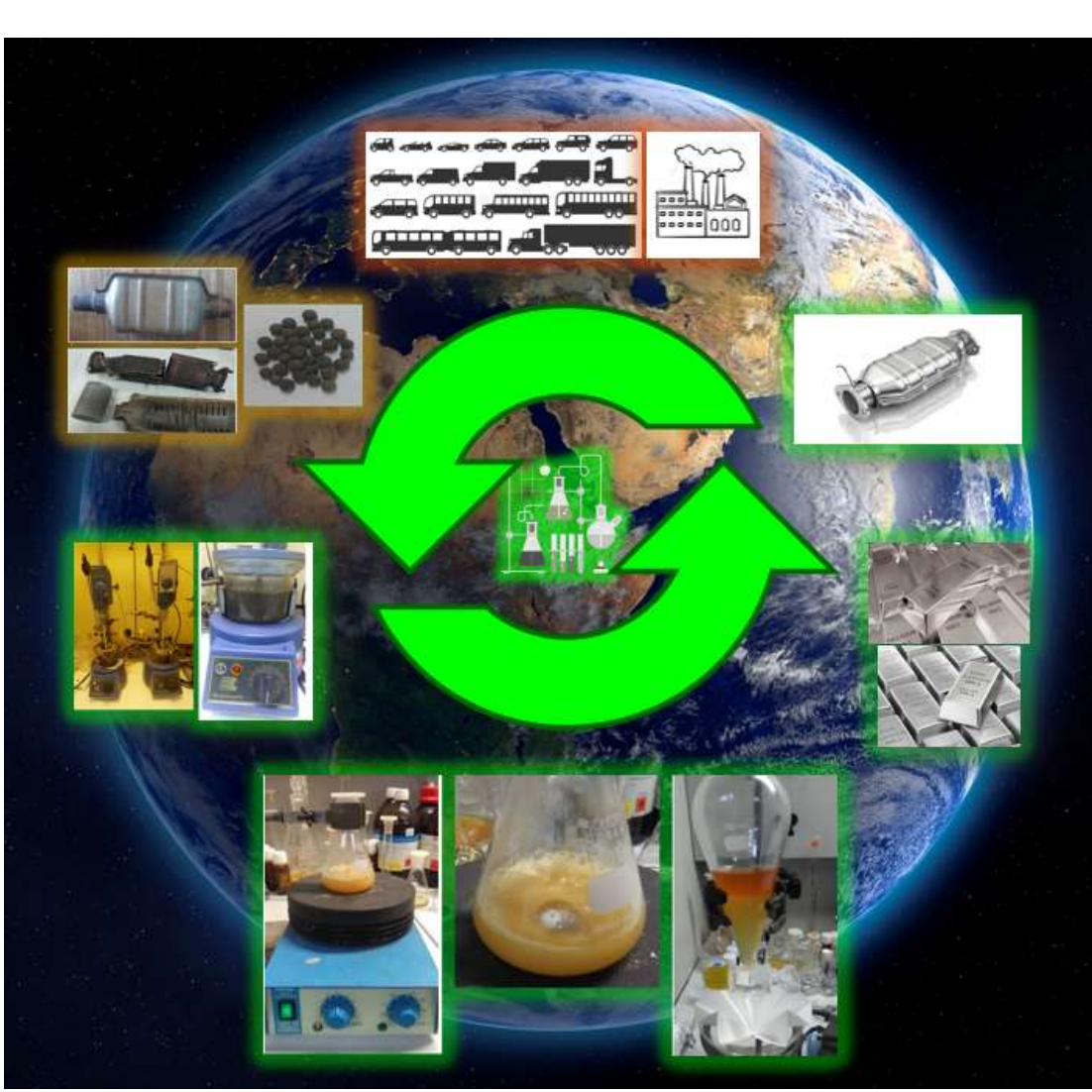

A relatively new technique, hydrometallurgy exploits aqueous chemistry to extract and purify metals. metals from end-of-life materials. One of the key ingredients of her approach is the design of new molecules that can act as efficient and selective extractan for metal recovery from waste catalysts. 'A few groups,' says Paiva, 'focus on the adjustment and optimisation of existing commercial extractants, whereas others preferto design new extracting to realise, however, that solutions

HYDROMETALLURGICAL SOLVENT EXTRACTION The development of integrated costThetive and environmentally friendly hydrometallurgical processes for platinumigroup metal recycling exploits a technique known as solvent extraction, or liquid-liquid extraction. In the first pa of this process, a metal-containing sample a strongly acidic or alkaline aqueous

Platinum-group metals are rare and precious resources of tremendous technological importance in electronics, chemistry, and pollution control.

obtained from anthropogenic samples generally exhibit different compositions compared to natural primary resources, and their treatment may therefore require different approaches.' Understanding how to syn hesise and tallor extactant molecules for specific metal recovery processes to be caried out on samples ofhuman, rather than natural, onigin is active, and tremendosy
field of investigation. solution is used to dissolve metal traces. In the case of platinum, palladium, and rhodium, for instance, hydrochloric acid is used for leaching, in preference to other chemicals like sulphuric acid or nitric acid. Chloride media are usually involved in platinum-group metals recovery, and preliminary investigation of solvent extraction processes should always be carried out prior to their leaching solutions. 'Many investigators are aware of the importance of applying successtul metal extraction schemes to Paiva. 'However, systematic fundamental studies are, in general, necessary and they are always crucial in assessing the actual potential of an extractant prior to

METAL ION TRANSFER

\section{Following the leaching, a solvent}

extraction scheme can then be applied

the extraction stage, the aqueous metal solution is put into contact with an organic solvent that can chemically interact with the aqueous metal species and form stable bonds with them, in the form of solvated ion pairs, for example. This is followed by a stripping phase, in which the metal-loaded organic solvent transfers the metal ions to a new aqueous solution from which the purfified metal can then be socolit Solate organic sect ions. The process is then repeated.

URBAN MINING

Successful solvent extraction processes 


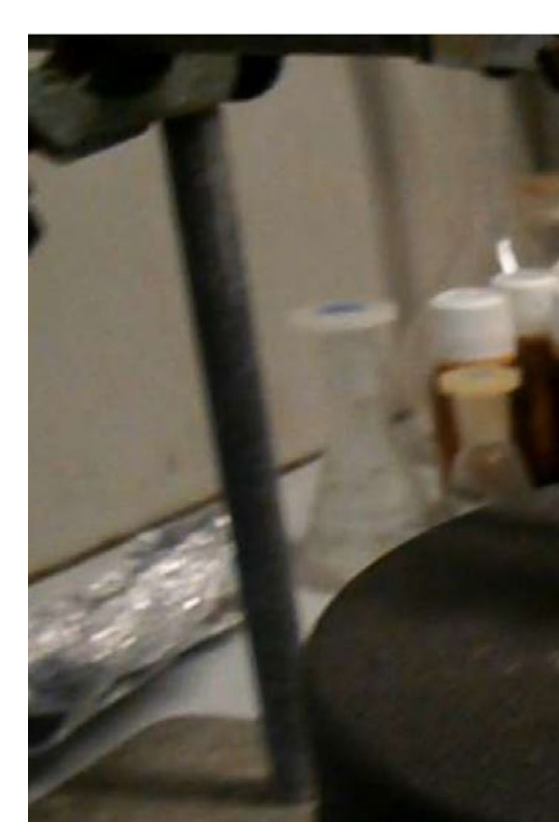

Optimising the solvent extraction process: Paive two extractants previously developed by esearch team.

can be used to separate the metal of interest from other impurities dissolved in the leaching solution. Additionally, the organic solvent can be reused in successive extraction-stripping cycles, and is not thought to suffer from chemica degradation during the extraction. Two components are vital for exploiting the stockpile of rare metals contained discarded electronic equipment and end-of-life catalytic materials: the ability to synthesise organic solvents that can

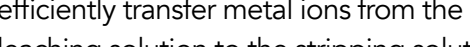
leaching solution to the stripping sol process for anthropogenic waste samples.

\section{OPTIMISING SOLVENT EXTRACTION} Understanding the factors affecting often requires a systematic assessment of the individual contributions to the solvent extraction process, including the role of the leaching solvent, the efficiency of the organic extractant and frequently, the influence of potential impurities. This is the first step towards optimising metal recovery for operation in realistic conditions. In a study of the recovery of palladium metal from spent industrial hydrogenation catalyss based on alumina (aluminium oxide) Paiva and her collaborators have systematically investigated he ability of

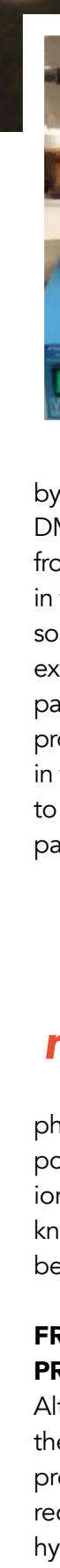

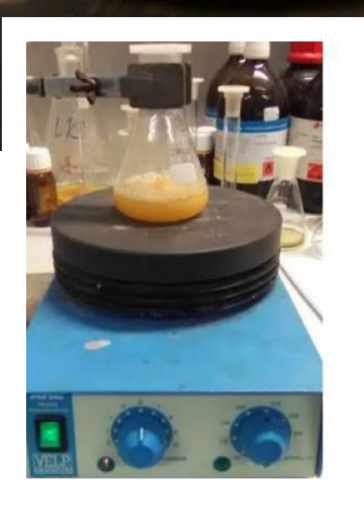

by her research team (MCHTA and DMDCHTDGA) to separate palladium from aluminium impurities co-dissolved in the hydrochloric acid-based leaching solution. Her results show that the in the organic solvation of aluminium

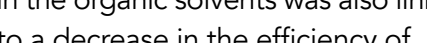
palladium recovery by the stripping

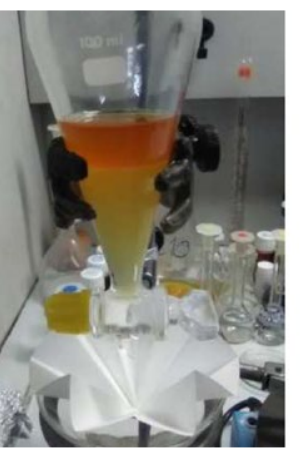

of metal waste is often affected by several complicating factors. One of the most ambitious goals of hydrometallurgy in urban mining is the recovery of platinum from spent automobile catalytic converters. In recent work, Paiva shows that one of the organic solvents used in her previous work on palladium recovery (DMDCHTCA) is also a promising medium for he extraction of platinum

Hydrometallurgy provides efficient routes to recycling end-of-life catalytic materials for the recovery of rare metals. phase. To address this complication, ions from the organic phases (a process known as scrubbing) are currently being investigated.

\section{FROM MODEL SOLUTIONS TO} PRACTICAL APPLICATIONS Although a careful optimisation of prerequisite for succesful ms a recovery, the practical application hydrometallurgy in the real-life treatment
However, its application to real catalytic converter samples proved to be far less successful. Although the organic phase exhibited an excellent loading performance for both platinum and iron (impurities Pans ions were not accomplished efficien Paiva's efforts are currently being directed toward testing the applicability presence of DMDCHTDGA. of solutions containing ammonia to
Research Objectives

Ana Paula Paiva's research applies solvent extraction to the concentration and/or purification of leaching solutions, particularly from the hydrometallurgical treatment of several end-of-life materials.

\section{Detail}

Centro de Química Estrutural (COE)

Institute of Molecular Sciences

Departamento de Quimica e Bioquimica

Canpo dande C8, 1749-016 Lisbo, Portigal

9-016 Lisboa, Portuga

Bio

Ana Paula Paiva has been Assistant Professor at

Departamento de Química e Bioquímica (DQB), Faculdade

de Ciências, Universidade de Lisboa (FCUL), since 1996.

She was awarded the Habilitation title in Chemistry by

member of Ce Lisboa in March 2021. She has been a

Funding

Fundação para a Ciência e a Tecnologia (FCT), IP/

MCTES, Lisbon, Portugal, through national funds

(PIDDAC) for the projects UIDMMULT/O06/2/2019,

UIDB/00100/2020, LA/P/0056/2020, and PTDC/BTA-

BTA/29251/2017. The latter project is co-financed by the

Algarve's Regional Operational Program (CRESC Algarve 2020), through Portugal 2020 and European Regional Development Fund (FEDER).

\section{Collaborators}

-CCMar, Universidade do Algarve,

Faro, Portugal

(Lebratório Nacional de Energia

Portug

Cape Verde

FCT
References

- Méndez, A, Nogueira, C, Paiva, A, (2021) Recovery of Platinum from a Spent Automotive Catalyst through Chloride Leaching and Solvent Extraction. Recycling, 6, 27. doi. org/10.3390/recycling6020027

- Paiva, A, (2021) Ana Paula Paiva. [online] Faculty of Sciences of the University of Lisbon. ciencias.ulisboa.pt/en/perfil/ appaiva [Accessed $07 \mathrm{Feb}$ 2022]

- Paiva, A, (2017) Recycling of Palladium from Spent Catalysts Using Solvent Extraction-Some Critical Points. Metals, 7, 505. www.mdpi.com/2075-4701/7/11/505

- Paiva, A, Ortet, O, Carvalho, G, Nogueira, C, (2017) Recovery of palladium from a spent industrial catalyst through leaching and solvent extraction. Hydrometallurgy, 171, 394-401. www.sciencedirect.com/science/article/abs/ pii//50304386X17300361?via\%3Dihub

- Ortet, O, Paiva, A, (2015) Liquid-liquid extraction of palladium(II) from chloride media by $N_{1} N^{\prime}$-dimethyl- $N, N^{\prime}$ dicyclohexylthiodiglycolamide. Separation and Purification Technology, 156, 363-368. www.sciencedirect.com/science/ article/abs/pii/S1383586615302744?via\%3Dihub - Ortet, O, Paiva, A, (2015) Development of tertiary thioamide derivatives to recover palladium (II) from simulated complex chloride solutions. Hydrometallurgy, 151, 33-41. www.sciencedirect.com/science/article/abs/piit S0304386X14002333?via\%3Dihub

\section{Personal Response}

What are the strengths and the current main limitations of hydrometallurgy in the recycling of platinum-group metals?

II As far as I know pyro- and hydrometallurgy are currently used together to process spent catalysts in most industrial facilities; the former is used for gross recovery, the latter for finer separations. The main strengths are the use of significantly lowertemperatures, potential to both sma-metal extraction, and the ability to operate at the abundance of pure scientific targets in literature (the applied sense is often put aside), and the lack of piloting or scaling-up investigation by the engineering community to evaluate the most promising hydrometallurgical processes already developed. 